\title{
Strong convergence of additive Multidimensional Continued Fraction algorithms
}

\author{
by \\ Kentaro NAKAishi (Tokyo)
}

1. Statements of results. By the paper of Lagarias [4], the metrical convergence of algorithms of simultaneous Diophantine approximation has been clearly formulated as a problem of ergodic theory and dynamical systems. Since then, much of the study was devoted to variants of the Jacobi-Perron algorithm, which is a direct generalisation of the continued fraction algorithm. There is another class of algorithms which includes the algorithms of Selmer and Brun. This class appears to be more practical since the operations of approximation consist of addition (subtraction) instead of multiplication as in the case of the Jacobi-Perron type algorithms. In this paper, we prove the almost everywhere strong convergence of a class of two-dimensional algorithms of additive type (Corollary 2). The problem of strong convergence concerns the speed of convergence of Diophantine quantities $q_{n} x-p_{n}$, which typically attenuate oscillating as $n$ goes to infinity. For future reference, we formulate our criterion for arbitrary finite-dimensional algorithms (Theorem 1).

Metric properties of Selmer's algorithm have already been studied by Schweiger [5]. Schweiger reduces Selmer's algorithm by the jump transformation to Baldwin's one, the ergodic properties of which are already established. However, his argument covers one particular sequence of fractions of approximation. Our method covers all the fractions given by the algorithm, which is complementary to Schweiger [5] in this respect, and as a consequence, we obtain information on the second Lyapunov exponent of the system.

For a proof of the strong convergence of two-dimensional Brun's algorithm for the multiplicative case (cf. Remark 1.1), we refer to BroiseAlamichel and Guivarc'h [1].

Definition 1.1. Let $d$ be a positive integer. A sequence of rational vectors in $\mathbb{R}^{d},\left(p_{n}^{(1)} / q_{n}, \ldots, p_{n}^{(d)} / q_{n}\right)$, is said to be weakly convergent to $\left(x_{1}, \ldots, x_{d}\right)$ if

2000 Mathematics Subject Classification: 11K55, 11J13, 11J70, 37A45, 37H15. 


$$
\lim _{n \rightarrow \infty}\left\|\left(x_{1}, \ldots, x_{d}\right)-\left(\frac{p_{n}^{(1)}}{q_{n}}, \ldots, \frac{p_{n}^{(d)}}{q_{n}}\right)\right\|=0
$$

where $\|\cdot\|$ denotes a fixed Euclidean norm in $\mathbb{R}^{d}$. If the rate of convergence is exponential, the sequence is called semi-weakly convergent. A sequence of rational vectors $\left(p_{n}^{(1)} / q_{n}, \ldots, p_{n}^{(d)} / q_{n}\right)$ is said to be strongly convergent to $\left(x_{1}, \ldots, x_{d}\right)$ if

$$
\lim _{n \rightarrow \infty}\left\|q_{n}\left(x_{1}, \ldots, x_{d}\right)-\left(p_{n}^{(1)}, \ldots, p_{n}^{(d)}\right)\right\|=0 .
$$

If the rate of convergence is exponential, the sequence is called exponentially strongly convergent.

For future use, we axiomatize our criterion for algorithms we consider to be strongly convergent. An additive Multidimensional Continued Fraction (MCF) algorithm in dimension $d$ is a triple $(T, \mu, M)$ satisfying the following conditions (H1)-(H5):

(H1) There is a Markovian dynamical system $T: \Delta \rightarrow \Delta$ such that (a) $\Delta$ is a compact connected $d$-dimensional manifold in $\mathbb{R}^{d}$ with piecewise smooth boundary, (b) $\Delta$ has a finite partition $\mathcal{P}$, each element of which has interior points, so that $T(\xi)$ is a (modulo 0 ) union of members of $\mathcal{P}$ for all $\xi \in \mathcal{P}$, (c) $T$ admits an invariant ergodic measure $\mu$ on $\Delta$ which is equivalent to Lebesgue measure, and (d) $T$ is piecewise continuous with non-vanishing Jacobian almost everywhere.

(H2) There exists a matrix-valued function $M: \Delta \rightarrow \mathrm{GL}(d+1,\{0,1\})$ which is constant on the interior of each element of $\mathcal{P}$ with $\int \log ^{+}\|M(p)\| d \mu$ $<\infty$.

Define a cocycle inductively by

$$
M_{n}(p)=M\left(T^{n-1}(p)\right) M_{n-1}(p)
$$

for $n \geq 1$, where $M_{0}$ is the identity matrix.

(H3) Set

$$
\tau(p)=\min \left\{k: M_{k}(p) \text { is strictly positive }\right\}
$$

and $\tau(p)=\infty$ if no such $k$ exists. Then

$$
\int \tau(p) d \mu<\infty
$$

Define

$$
\left(\begin{array}{cccc}
p_{n}^{(0,1)} & \cdots & p_{n}^{(0, d)} & q_{n}^{(0)} \\
p_{n}^{(1,1)} & \cdots & p_{n}^{(1, d)} & q_{n}^{(1)} \\
\vdots & & \vdots & \vdots \\
p_{n}^{(d, 1)} & \cdots & p_{n}^{(d, d)} & q_{n}^{(d)}
\end{array}\right):=M_{n}(p)
$$


A MCF algorithm is said to be weakly convergent at $p$ if all the convergents

$$
\left(\frac{p_{n}^{(j, 1)}}{q_{n}^{(j)}}, \ldots, \frac{p_{n}^{(j, d)}}{q_{n}^{(j)}}\right)
$$

for $0 \leq j \leq d$ are weakly convergent at $p$; it is called strongly convergent if all the convergents are strongly convergent.

(H4) The algorithm is weakly convergent at $\mu$-almost every point $p \in \Delta$.

Definition 1.2. A real matrix $M$ is spectrally $P V$ (Pisot-Vijayaraghavan) if there is a simple (and hence real) eigenvalue $|\lambda|>1$ of $M$ such that all other eigenvalues are strictly less than one in modulus.

(H5) There exists an element $\xi_{0}$ of $\mathcal{P}$ with $\xi_{0} \subset T\left(\xi_{0}\right)$ so that $M(p)$ is spectrally $\mathrm{PV}$ on the interior of $\xi_{0}$ with a positive eigenvector for the simple eigenvalue $|\lambda|>1$.

Remark 1.1. Conditions (H1)-(H5) include the notion of additive algorithm in the sense of Lagarias [4], where an additive MCF algorithm is characterised by the fact that the number of allowable associated matrices $M(p)$ is finite. The Jacobi-Perron type algorithms are multiplicative since it is infinite.

Given $p=\left(x_{1}, \ldots, x_{d}\right) \in \Delta$, define

$$
\begin{aligned}
D_{n}^{(j, i)} & =q_{n}^{(j)} x_{i}-p_{n}^{(j, i)} & & \text { for } 0 \leq j \leq d \text { and } 1 \leq i \leq d, \\
\varrho_{n}^{(i)} & =\max _{0 \leq j \leq d}\left\{\left|D_{n}^{(j, i)}\right|\right\} & & \text { for } 1 \leq i \leq d .
\end{aligned}
$$

(H6) For each $i, 1 \leq i \leq d$, there exists an integer $l>0$ so that for almost every point $p$ one can find a subsequence $\left\{n_{k}\right\}$ and a constant $C_{1}>0$ with the following properties:

(a) $\varrho_{n_{k+1}}^{(i)} \leq \varrho_{n_{k}}^{(i)}$ for all $k \geq 1$,

(b) $\varrho_{n_{k}+j}^{(i)} \leq C_{1} \varrho_{n_{k}}^{(i)}$ for $1 \leq j \leq n_{k+1}-n_{k}-1$ and all $k \geq 1$,

(c) if $T^{j}(p), T^{j+1}(p), \ldots, T^{j+n l-1}(p) \in \xi_{0}$ for $n \geq 1$, then

$$
\#\left\{\left\{n_{k}\right\}_{k \geq 1} \cap\{j, j+1, \ldots, j+n l-1\}\right\} \geq n .
$$

TheOREm 1. Assume that an additive $M C F$ algorithm $(T, \mu, M)$ in dimension $d$ satisfies condition (H6). Then the algorithm is exponentially strongly convergent for almost every point $p \in \Delta$. Furthermore, the second Lyapunov exponent of the system $(T, \mu, M)$ is strictly negative.

In [4] the algorithms of Brun and Selmer have been verified to be additive MCF algorithms in our sense.

COROLlary 2. The algorithms of Brun and Selmer in dimension two satisfy condition $(\mathrm{H} 6)$. 
2. Proof of Theorem 1 and Corollary 2. For a target vector $p=$ $\left(x_{1}, \ldots, x_{d}\right)$, an additive MCF algorithm in dimension $d$ provides convergents

$$
\mathcal{C}_{n}\left(x_{i}\right)=\left(\frac{p_{n}^{(0, i)}}{q_{n}^{(0)}}, \frac{p_{n}^{(1, i)}}{q_{n}^{(1)}}, \ldots, \frac{p_{n}^{(d, i)}}{q_{n}^{(d)}}\right)
$$

for $n \geq 1$ and $1 \leq i \leq d$. We say that the status of a convergent $p_{n}^{(j, i)} / q_{n}^{(j)}$ for $x_{i}$ is $j$. Notice that

(1) $\quad\left(\begin{array}{cccc}p_{n}^{(0,1)} & \cdots & p_{n}^{(0, d)} & q_{n}^{(0)} \\ \vdots & & \vdots & \vdots \\ p_{n}^{(d, 1)} & \cdots & p_{n}^{(d, d)} & q_{n}^{(d)}\end{array}\right)=M\left(T^{n-1}(p)\right)\left(\begin{array}{cccc}p_{n-1}^{(0,1)} & \cdots & p_{n-1}^{(0, d)} & q_{n-1}^{(0)} \\ \vdots & & \vdots & \vdots \\ p_{n-1}^{(d, 1)} & \cdots & p_{n-1}^{(d, d)} & q_{n-1}^{(d)}\end{array}\right)$.

Multiplying by the matrix

$$
\left(\begin{array}{ccccc}
-1 & 0 & \cdots & 0 & 0 \\
0 & -1 & \cdots & 0 & 0 \\
\vdots & \vdots & & \vdots & \vdots \\
0 & 0 & \cdots & -1 & 0 \\
x_{1} & x_{2} & \cdots & x_{d} & -1
\end{array}\right)
$$

from the right, we obtain

$$
v_{n}^{(i)}=M\left(T^{n-1}(p)\right) v_{n-1}^{(i)}
$$

for $1 \leq i \leq d$, where

$$
v_{n}^{(i)}:=\left(\begin{array}{c}
D_{n}^{(0, i)} \\
\vdots \\
D_{n}^{(d, i)}
\end{array}\right)
$$

Notice that $\varrho_{n}^{(i)}$ is the maximal norm of $v_{n}^{(i)}$. The problem of strong convergence is to show how the vectors $v_{n}^{(i)}$ are attracted to zero under the "random" matrix (2). Equivalently, it is the problem to show how all $\varrho_{n}^{(i)}$ converge to zero.

Lemma 3. Let $a, b, c$ and $d$ be positive real numbers with $a d-b c \leq 0$. Then

$$
\frac{a}{b} \leq \frac{a+c}{b+d} \leq \frac{c}{d} .
$$

Proof. Simply compute.

Define a special cone domain in $\mathbb{R}^{d+1}$ by

$$
\Lambda_{d+1}=\left\{\left(x_{i}\right) \in \mathbb{R}^{d+1}: x_{i} \geq 0 \forall i\right\} \cup\left\{\left(x_{i}\right) \in \mathbb{R}^{d+1}: x_{i} \leq 0 \forall i\right\} .
$$


LEMma 4. If the additive algorithm is weakly convergent at $p$, then the sequences of vectors $v_{n}^{(i)}$ never fall into $\Lambda_{d+1}$ for any $n$ and $1 \leq i \leq d$.

Proof. Fix $i$. Suppose that $v_{n}^{(i)}$ falls into $\Lambda_{d+1}$ for some $n$. Then all the convergents

$$
p_{n}^{(0, i)} / q_{n}^{(0)}, \ldots, p_{n}^{(d, i)} / q_{n}^{(d)}
$$

lie on the same side of $x_{i}$ on the real line. Since $M(p)$ is a $0-1$ matrix by (H2) and since all the convergents at time $n+1$ are linear combinations of the convergents at time $n$ by (1), it follows from Lemma 3 that all the convergents after time $n$ remain in the convex hull (interval or point) spanned by (3). Thus they never approach $x_{i}$, which means there is no weak convergence.

Lemma 5. Let $A$ be a spectrally $P V(d+1) \times(d+1)$ matrix which has a positive eigenvector for the simple eigenvalue $|\lambda|>1$. Then there exists a uniform constant $0<\alpha<1$ such that if $v$ and $A^{m} v$ stay in the complement of the cone neighbourhood $\Lambda_{d+1}$ for sufficiently large $m$, then

$$
\left\|A^{m} v\right\| \leq \alpha\|v\| \text {. }
$$

Proof. Order the eigenvalues of $A$ in modulus as

$$
|\lambda|=\left|\lambda_{1}\right|>1>\left|\lambda_{2}\right| \geq\left|\lambda_{3}\right| \geq \cdots \geq\left|\lambda_{d+1}\right| .
$$

Denote the projection of $v$ onto the expanding eigenspace and its complementary subspace by $v_{\mathrm{e}}$ and $v_{\mathrm{c}}$ respectively. Then $v=v_{\mathrm{e}}+v_{\mathrm{c}}$ and $A v=\lambda_{1} v_{\mathrm{e}}+A v_{\mathrm{c}}$. It is obvious that there is a uniform constant $\delta>0$ such that $\left\|p_{\mathrm{e}}\right\| \leq \delta\left\|p_{\mathrm{c}}\right\|$ for any vector $p=p_{\mathrm{e}}+p_{\mathrm{c}} \notin \Lambda_{d+1}$. Notice that $A$ can be decomposed, via a suitable invertible matrix $P$, into diagonal parts and nilpotent parts:

$$
P^{-1} A P=\lambda_{1} E_{1}+\sum_{j=2}^{D}\left(\lambda_{j} E_{j}+N_{j}\right)
$$

where $E_{j}$ and $N_{j}$ stand respectively for the identity and the nilpotent part on the generalised eigenspace associated with $\lambda_{j}$, and $D \leq d+1$. Since

$$
\left(\lambda_{j} E_{j}+N_{j}\right)^{n}=\lambda_{j}^{n} E_{j}+\left(\begin{array}{c}
n \\
1
\end{array}\right) \lambda_{j}^{n-1} N_{j}+\cdots+\left(\begin{array}{c}
n \\
l
\end{array}\right) \lambda_{j}^{n-l} N_{j}^{l}
$$

for some $l$, one can find an integer $k$ so that

$$
\alpha:=(\delta+1) D \max _{2 \leq j \leq D}\left\{\left\|P\left(\lambda_{j} E_{j}+N_{j}\right)^{k} P^{-1}\right\|\right\}<1 .
$$

Here $\|B\|$ denotes the operator norm of a matrix $B$. Suppose that the hypothesis is valid for $m \geq k$. Then we obtain

$$
\left\|A^{m} v\right\| \leq(\delta+1)\left\|A^{m} v_{\mathrm{c}}\right\| \leq(\delta+1) \sum_{j=2}^{D}\left\|P\left(\lambda_{j} E_{j}+N_{j}\right)^{m} P^{-1} v_{\mathrm{c}}\right\| \leq \alpha\|v\| .
$$


It does not matter which norm is taken since we can take $\alpha$ as small as we want, if necessary.

REMARK 2.1. The idea of the proof of Lemma 5 is based on the elementary facts of the dynamical system theory. See [3] for example. Remember the behaviour of the hyperbolic flow $\exp (t A) v$ on $\mathbb{R}^{d+1}$. If the orbit stays away from a neighbourhood $\Lambda_{d+1}$ of the expanding direction, then it necessarily gets contracted.

Proof of Theorem 1. For each $i$, we will find a constant $0<\gamma<1$ so that for almost every $p=\left(x_{1}, \ldots, x_{d}\right) \in \Delta$ there exists an integer $n(p)$ with

$$
\varrho_{n}^{(i)} \leq \gamma^{n} \quad \text { for all } n \geq n(p) .
$$

We may assume by (H4) that the algorithm is weakly convergent at $p$.

By (H1), the dynamics of $T$ can be described by a subshift of finite type through a Markov partition $\mathcal{P}$. We have $\mu(\xi)>0$ for all $\xi \in \mathcal{P}$ because $\mu$ detects interior points by (H1)(c). Moreover, by (H1)(d) and (H5),

$$
\Gamma_{n}:=\xi_{0} \cap T^{-1} \xi_{0} \cap \cdots \cap T^{-(n-1)} \xi_{0}
$$

has a positive $\mu$-measure for every $n \geq 1$.

Observe that, by Birkhoff's ergodic theorem,

$$
\lim _{n \rightarrow \infty} \frac{1}{n} \sum_{k=0}^{n-1} \chi_{E}\left(T^{k}(p)\right)=\mu(E) \quad \mu \text {-a.e. } p
$$

where $\chi_{E}$ denotes the indicator function of a measurable set $E$.

The orbit makes a consecutive visit of length $i$ to $\xi_{0}$ if

$$
T^{j-1}(p) \notin \xi_{0}, \quad T^{j}(p), T^{j+1}(p), \ldots, T^{j+i-1}(p) \in \xi_{0}, \quad T^{j+i}(p) \notin \xi_{0}
$$

for some $j$. Define an $l$-block to be the event that

$$
T^{j}(p), T^{j+1}(p), \ldots, T^{j+l-1}(p) \in \xi_{0}
$$

for some $j$. We view a consecutive visit of length $i$ as concatenation of $[i / l] l$-blocks plus a residual block, where $[x]$ denotes the integral part of a real number $x$. For example, we regard a consecutive visit of length $2 l$ as concatenation of two $l$-blocks, while a consecutive visit of length $2 l-1$ as one $l$-block plus a residual block.

Set $\Gamma:=\Gamma_{l}$ for $l$ in (H6). Suppose that we have $L$ consecutive visits in the orbit $\mathcal{O}=\left\{p, T(p), \ldots, T^{n_{k}-1}(p)\right\}$ with lengths $i_{1}, \ldots, i_{L}$. Notice that $T^{n_{k}-1}(p)$ may be in the middle of the last consecutive visit of length $i_{L}$. In that case, we pretend that the last consecutive visit to $\xi_{0}$ ends at $T^{n_{k}-1}(p)$ and reset $i_{L}$. Then the number of $l$-blocks in the orbit $\mathcal{O}$ is equal to

$$
\sum_{j=1}^{L}\left[\frac{i_{j}}{l}\right]
$$


Since

$$
\sum_{j=0}^{n_{k}-1} \chi_{\Gamma}\left(T^{j}(p)\right)=\sum_{j=1}^{L} \max \left\{i_{j}-l+1,0\right\},
$$

(H6)(c) implies

$$
\frac{k}{n_{k}} \geq \frac{1}{n_{k}} \sum_{j=1}^{L}\left[\frac{i_{j}}{l}\right] \geq \frac{1}{n_{k}}\left(\frac{1}{l} \sum_{j=0}^{n_{k}-1} \chi_{\Gamma}\left(T^{j}(p)\right)\right) .
$$

Thus, by (4) and $\mu(\Gamma)>0$, there are a uniform constant $0<\gamma_{1}<1$ and an integer $k(p)$ such that $n_{k} \geq k \geq \gamma_{1} n_{k}$ for all $k \geq k(p)$ (a.e. $p$ ).

On the other hand, if $T^{j}(p) \in \xi_{0}$ for $a \leq j \leq a+b-1$, then $M\left(T^{j}(p)\right)=A$ for $a \leq j \leq a+b-1$, where $A$ is a spectrally PV matrix. Since the algorithm is weakly convergent at $p$, Lemma 4 holds. Thus we can apply Lemma 5 to $v^{(i)}$ via (2). Lemma 5 implies that there are a constant $0<\alpha<1$ and an integer $m>0$ such that if $T^{j}(p) \in \xi_{0}$ for $a \leq j \leq a+m-1$, then $\varrho_{a+m}^{(i)} \leq \alpha \varrho_{a}^{(i)}$.

Put $N=([m / l]+3) l$. A consecutive visit of length $N$ includes at least $[m / l]+3$ members of the subsequence $\left\{n_{k}\right\}$. The difference of the beginning and ending of these members in this consecutive visit is more than $m$. Thus we can take a sub-subsequence $\left\{n_{k_{b}}\right\}$ so that

$$
\varrho_{n_{k_{b+1}}}^{(i)} \leq \alpha \varrho_{n_{k_{b}}}^{(i)} \quad \text { if } b \text { is odd }
$$

We use the property $\varrho_{n_{k+1}}^{(i)} \leq \varrho_{n_{k}}^{(i)}$ if $b$ is even. Considering the number of $N$-blocks, we obtain

$$
\frac{b}{n_{k_{b}}} \geq \frac{2}{n_{k_{b}}}\left(\frac{1}{N} \sum_{a=0}^{n_{k_{b}}-1} \chi_{\Gamma_{N}}\left(T^{a}(p)\right)\right) .
$$

By (4) and $\mu\left(\Gamma_{N}\right)>0$, there are a uniform constant $0<\gamma_{2}<1$ and an integer $b(p)$ such that $2 n_{k_{b}} \geq b \geq 2 \gamma_{2} n_{k_{b}}$ for all $b \geq b(p)$ (a.e. $p$ ). Combining this with (5), we have

$$
\varrho_{n_{k_{b}}}^{(i)} \leq \exp (-\beta b) \leq \exp \left(-2 \beta \gamma_{2} n_{k_{b}}\right)
$$

for some $\beta>0$ and all $b \geq 1$. Since

$$
\frac{n_{k_{b}+j}}{n_{k_{b}}} \leq \frac{n_{k_{b+1}}}{n_{k_{b}}} \leq \frac{b+1}{b} \frac{b}{n_{k_{b}}} \frac{n_{k_{b+1}}}{b+1} \leq \frac{3}{4 \gamma_{2}}
$$

for $1 \leq j \leq k_{b+1}-k_{b}-1$, there exist $\gamma_{3}>0$ and an integer $k_{1}(p)>0$ so that

$$
\varrho_{n_{k}}^{(i)} \leq \exp \left(-\gamma_{3} n_{k}\right) \quad \text { for all } k \geq k_{1}(p)
$$


Now (H6)(b) implies that for $1 \leq j \leq n_{k+1}-n_{k}-1$,

$$
\begin{aligned}
\varrho_{n_{k}+j}^{(i)} \leq C_{1} \exp \left(-\gamma_{3} n_{k}\right) & =C_{1} \exp \left(-\frac{\gamma_{3} n_{k}}{2}\right) \exp \left(-\frac{\gamma_{3} n_{k}}{2}\right) \\
& \leq \exp \left(-\frac{3 \gamma_{3}}{4 \gamma_{1}}\left(n_{k}+j\right)\right)
\end{aligned}
$$

since

$$
\frac{n_{k}+j}{n_{k}} \leq \frac{n_{k+1}}{n_{k}} \leq \frac{k+1}{k} \frac{k}{n_{k}} \frac{n_{k+1}}{k+1} \leq \frac{3}{2 \gamma_{1}}
$$

for all $k \geq k_{1}(p)$ (resetting $k_{1}(p)$ if necessary).

Notice that $\varrho_{n} \leq \gamma^{n}$ for some $0<\gamma<1$ and $1 \leq i \leq d$ imply the semi-weak convergence, if only $q_{n}^{(j)}>0$ for some $j$. Using the argument in [4], we have

$$
\lim _{n \rightarrow \infty} \frac{1}{n} \log q_{n}^{(j)}=\lambda_{1} \quad \text { a.e. }
$$

for $0 \leq j \leq d$, where $\lambda_{1}>0$ is the first Lyapunov exponent. As a consequence, there exists $\delta>1$ so that for almost every point $p$ one can find an integer $N(p)>0$ so that

$$
\left|x_{i}-\frac{p_{n}^{(j, i)}}{q_{n}^{(j)}}\right| \leq \frac{1}{\left(q_{n}^{(j)}\right)^{\delta}}
$$

for all $n \geq N(p), 1 \leq i \leq d$ and $0 \leq j \leq d$.

Let $\lambda_{2}$ be the second Lyapunov exponent of the algorithm $(T, \mu, M)$. Then it is obvious that $\lambda_{2}<0$ since

$$
\sum_{i=1}^{d} \sum_{j=0}^{d}\left|q_{n}^{(j)} x_{i}-p_{n}^{(j, i)}\right|=\exp \left(\lambda_{2} n(1+o(1))\right)
$$

(see (4.21) in [4]).

In the following sections, condition (H6) will be verified for the algorithms of Selmer and Brun in dimension two.

3. The algorithm of Selmer [7]. Consider a three-dimensional simplex

$$
\Omega^{2+1}=\left\{\mathbf{b}=\left(b_{1}, b_{2}, b_{3}\right): 0 \leq b_{1} \leq b_{2} \leq b_{3}\right\} .
$$

Define a transformation $\widetilde{T}: \Omega^{2+1} \rightarrow \Omega^{2+1}$ obtained from

$$
\sigma \mathbf{b}=\left(b_{1}, b_{2}, b_{3}-b_{1}\right)
$$

by making necessary permutations of coordinates to have the image in $\Omega^{2+1}$. Finally, a transformation $T: \Delta^{\prime} \rightarrow \Delta^{\prime}$ is obtained by a commutative diagram $T \circ \pi=\pi \circ \widetilde{T}$, where $\pi\left(b_{1}, b_{2}, b_{3}\right)=\left(b_{1} / b_{3}, b_{2} / b_{3}\right)$ is the quotient map and

$$
\Delta^{\prime}=\{(x, y): 0 \leq x \leq y \leq 1\} .
$$


There is a natural partition

$$
\begin{aligned}
\Delta^{\prime}= & \Delta(0) \cup \Delta(1) \cup \Delta(2) \\
= & \{(x, y) \in \Delta: x+y \leq 1\} \cup\{(x, y) \in \Delta: x \geq 1 / 2\} \\
& \cup\{(x, y) \in \Delta: x+y \geq 1, x \leq 1 / 2\}
\end{aligned}
$$

such that

$$
T(x, y)= \begin{cases}\left(\frac{x}{1-x}, \frac{y}{1-x}\right) & \text { if }(x, y) \in \Delta(0), \\ \left(\frac{1-x}{y}, \frac{x}{y}\right) & \text { if }(x, y) \in \Delta(1), \\ \left(\frac{x}{y}, \frac{1-x}{y}\right) & \text { if }(x, y) \in \Delta(2) .\end{cases}
$$

Since $T(\Delta(0))=\Delta^{\prime}$ and $T(\Delta(j))=\Delta(1) \cup \Delta(2)$ (injectively onto) for $j=1$ and 2, the map $T$ is transient on $\Delta(0)$. Thus one needs to consider the dynamical system $T: \Delta \rightarrow \Delta$ on the invariant domain $\Delta:=\Delta(1) \cup \Delta(2)$. Set $\left(x_{n}, y_{n}\right)=T^{n}(x, y)$ for $n>0$ and $\left(x_{0}, y_{0}\right)=(x, y)$. Let

$$
\varepsilon(x, y)= \begin{cases}1 & \text { if }(x, y) \in \Delta(1), \\ 2 & \text { if }(x, y) \in \Delta(2) .\end{cases}
$$

Define a coding $\left\{\varepsilon_{n}\right\}$ of the orbit by $\varepsilon_{n}=\varepsilon\left(x_{n}, y_{n}\right)$ and $\varepsilon_{0}=\varepsilon(x, y)$. Then, using the row vector $u_{1}=\left(x_{1}, y_{1}, 1\right)$, the equation

$$
u_{0}=\theta(x, y) u_{1} M(x, y)
$$

defines the associated $3 \times 3$ matrices $M(x, y)$, where $\theta(x, y)=1 /\left(x_{1}+y_{1}\right)$. This paper adopts the same matrix representation as in [4]. Often the transposed matrix is used.

Two-dimensional Selmer's algorithm has only two matrices allowable:

$$
M(x, y)=\left(\begin{array}{lll}
0 & 0 & 1 \\
1 & 0 & 1 \\
0 & 1 & 0
\end{array}\right) \quad \text { if } \varepsilon_{0}=1, \quad M(x, y)=\left(\begin{array}{lll}
1 & 0 & 1 \\
0 & 0 & 1 \\
0 & 1 & 0
\end{array}\right) \quad \text { if } \varepsilon_{0}=2
$$

Then two recursive equations are obtained:

$$
\begin{aligned}
& q_{n+1}^{(0)}=q_{n}^{(2)}, \quad q_{n+1}^{(1)}=q_{n}^{(0)}+q_{n}^{(2)}, \quad q_{n+1}^{(2)}=q_{n}^{(1)} \quad \text { if } \varepsilon_{n}=1, \\
& q_{n+1}^{(0)}=q_{n}^{(0)}+q_{n}^{(2)}, \quad q_{n+1}^{(1)}=q_{n}^{(2)}, \quad q_{n+1}^{(2)}=q_{n}^{(1)} \quad \text { if } \varepsilon_{n}=2 .
\end{aligned}
$$

If we set $p_{n}^{(j)}:=p_{n}^{(j, 1)}$ and $r_{n}^{(j)}:=p_{n}^{(j, 2)}$, then the same recursive equations hold for $p_{n}^{(j)}$ and $r_{n}^{(j)}$. The following facts are well known (see [4], [6] for example):

- $\mu$ is ergodic and has an invariant density $d x d y / x y$.

- Selmer's algorithm is weakly convergent at almost every point in $\Delta$. 
And it is easy to see that

- $M(x, y)$ is spectrally $\mathrm{PV}$ on $\Delta(1)$ with a positive eigenvector.

We will verify (H6) only for $\varrho_{n}^{(2)}$. Similar results for $\varrho_{n}^{(1)}$ can be easily obtained. Set $D_{n}^{(j)}:=D_{n}^{(j, 2)}=q_{n}^{(j)} y-r_{n}^{(j)}$ and $\varrho_{n}:=\varrho_{n}^{(2)}=\max _{0 \leq j \leq 2}\left\{\left|D_{n}^{(j)}\right|\right\}$.

Definition 3.1. A triple of convergents $\mathcal{C}_{n}=\left(r_{n}^{(0)} / q_{n}^{(0)}, r_{n}^{(1)} / q_{n}^{(1)}, r_{n}^{(2)} / q_{n}^{(2)}\right)$ at time $n$ is said to be balanced for $y$ if

$$
\left|D_{n}^{(j)}\right| \geq\left|D_{n}^{(k)}\right| \geq\left|D_{n}^{(l)}\right|, \quad\{j, k, l\}=\{0,1,2\},
$$

and $y$ lies between $r_{n}^{(j)} / q_{n}^{(j)}$ and $r_{n}^{(k)} / q_{n}^{(k)}$ on the real line. We use the convention that $0 / 0=0$ and $1 / 0=\infty$.

Let

$$
(A, B, C)=\left(\frac{r_{n}^{(0)}}{q_{n}^{(0)}}, \frac{r_{n}^{(1)}}{q_{n}^{(1)}}, \frac{r_{n}^{(2)}}{q_{n}^{(2)}}\right) .
$$

Definition 3.2. Any triple $\mathcal{C}_{n}=(A, B, C)$ has a constellation which belongs to one of the following types:

(G1) $\quad A \leq y \leq B$ and $y \leq C \quad$ (or $A \geq y \geq B$ and $y \geq C$ ).

(G2) $\quad A \leq y \leq C$ and $B \leq y \quad$ (or $A \geq y \geq C$ and $B \geq y$ ).

(B) $\quad B \leq y \leq C$ and $y \leq A$ (or $B \geq y \geq C$ and $y \geq A$ ).

If $\mathcal{C}_{n}$ is of type (B), it is said to have a bad constellation. Otherwise $\mathcal{C}_{n}$ is said to have a good constellation.

Lemma 6. Suppose that the algorithm is weakly convergent at $(x, y)$. The following cases never happen to $\mathcal{C}_{n}$ :

(a) $\mathcal{C}_{n}$ is of type (G1) with $\left|D_{n}^{(2)}\right| \geq\left|D_{n}^{(0)}\right|$.

(b) $\mathcal{C}_{n}$ is of type (G2) with $\left|D_{n}^{(0)}\right| \geq\left|D_{n}^{(2)}\right|$ and $\left|D_{n}^{(1)}\right| \geq\left|D_{n}^{(2)}\right|$.

(c) $\mathcal{C}_{n}$ is of type (B) with $\left|D_{n}^{(2)}\right| \geq\left|D_{n}^{(1)}\right|$.

(d) $\mathcal{C}_{n}$ is of type (G1) with $\left|D_{n}^{(1)}\right| \geq\left|D_{n}^{(0)}\right| \geq\left|D_{n}^{(2)}\right|$.

Proof. Suppose (a). We may assume that $A \leq y \leq B$ and $y \leq C$. If $\varepsilon_{n}=1$, then

$$
D_{n+1}^{(0)}=D_{n}^{(2)}, \quad D_{n+1}^{(1)}=D_{n}^{(0)}+D_{n}^{(2)}, \quad D_{n+1}^{(2)}=D_{n}^{(1)}
$$

and if $\varepsilon_{n}=2$, then

$$
D_{n+1}^{(0)}=D_{n}^{(0)}+D_{n}^{(2)}, \quad D_{n+1}^{(1)}=D_{n}^{(2)}, \quad D_{n+1}^{(2)}=D_{n}^{(1)} .
$$

Since $D_{n}^{(2)}+D_{n}^{(0)}<0$, all the convergents at time $n+1$ are larger than $y$. Hence there is no weak convergence by Lemma 4. Consequently, (a) never happens. 
Suppose (b). We may assume that $C \leq y \leq A$ and $y \leq B$. If $\varepsilon_{n}=1$, then the constellation at time $n+1$ is of type (G1) with $\left|D_{n+1}^{(0)}\right| \leq\left|D_{n+1}^{(2)}\right|$, which satisfies (a), and hence this case is impossible. If $\varepsilon_{n}=2$, then the next constellation is of type (B) with $\left|D_{n+1}^{(2)}\right| \geq\left|D_{n+1}^{(1)}\right|$. Thus, whichever value $\varepsilon_{n+1}$ takes, the constellation at time $n+2$ satisfies (b) again. Repeating such return to (b) implies that the convergent $C$ remains throughout the whole process, though its status changes. This contradicts the weak convergence. Consequently, (b) never happens as well.

Suppose (c). We may assume that $B \leq y \leq A$ and $y \leq C$. Whichever value $\varepsilon_{n}$ takes, $\mathcal{C}_{n+1}$ has a constellation which satisfies (b). Hence (c) never appears.

Suppose (d). We may assume that $A \leq y \leq B$ and $y \leq C$. If $\varepsilon_{n}=1$, then $\mathcal{C}_{n+1}$ has a constellation which satisfies (c). If $\varepsilon_{n}=2$, then $\mathcal{C}_{n+1}$ has a constellation which satisfies (a). Hence (d) never happens.

Lemma 7. Suppose that the algorithm is weakly convergent at $(x, y)$.

(a) The triples of convergents $\mathcal{C}_{n}=\left(r_{n}^{(0)} / q_{n}^{(0)}, r_{n}^{(1)} / q_{n}^{(1)}, r_{n}^{(2)} / q_{n}^{(2)}\right)$ generated by the algorithm are balanced for all $n$.

(b) For any integer $k$, four consecutive triples $\left(\mathcal{C}_{k}, \mathcal{C}_{k+1}, \mathcal{C}_{k+2}, \mathcal{C}_{k+3}\right)$ with $\varepsilon_{k+j}=1(0 \leq j \leq 3)$ include at least one constellation of type (B).

Proof. The proof proceeds by induction and dividing into cases. It is easy to verify that $\mathcal{C}_{1}$ is balanced. Suppose that a triple of convergents $\mathcal{C}_{n}$ at time $n$ is balanced.

CASE I: $\left|D_{n}^{(2)}\right| \geq\left|D_{n}^{(0)}\right| \geq\left|D_{n}^{(1)}\right|$. Being balanced implies that $y$ lies between $A$ and $C$. Possible constellations are therefore (G1) and (G2). Since the constellation of type (G1) is prohibited by Lemma 6(a), the possible constellation at time $n$ is of type (G2). If $\varepsilon_{n}=1$, then the constellation at time $n+1$ is of type (G2) with $\varrho_{n+1}=\left|D_{n+1}^{(0)}\right|$. For balance at time $n+1$, the inequality

$$
\left|D_{n+1}^{(1)}\right|=\left|D_{n}^{(0)}+D_{n}^{(2)}\right| \leq\left|D_{n+1}^{(2)}\right|=\left|D_{n}^{(1)}\right|
$$

must hold, for if not, Lemma 6 (b) holds for $\mathcal{C}_{n+1}$, contrary to weak convergence. Thus $\mathcal{C}_{n+1}$ must be balanced with

$$
\left|D_{n+1}^{(0)}\right| \geq\left|D_{n+1}^{(2)}\right| \geq\left|D_{n+1}^{(1)}\right| \quad(\rightarrow \text { Case V }(\mathrm{G} 2)) .
$$

If $\varepsilon_{n}=2$, then the constellation at time $n+1$ is of type (G2) with $\varrho_{n+1}=$ $\left|D_{n+1}^{(1)}\right|$. For balance at time $n+1$, the inequality

$$
\left|D_{n+1}^{(0)}\right|=\left|D_{n}^{(0)}+D_{n}^{(2)}\right| \leq\left|D_{n+1}^{(2)}\right|=\left|D_{n}^{(1)}\right|
$$


must hold, for if not, Lemma 6 (b) holds for $\mathcal{C}_{n+1}$. Thus $\mathcal{C}_{n+1}$ must be balanced.

CASE II: $\left|D_{n}^{(2)}\right| \geq\left|D_{n}^{(1)}\right| \geq\left|D_{n}^{(0)}\right|$. Being balanced implies that $y$ lies between $B$ and $C$. Possible constellations are therefore (B) and (G2). Since the constellation of type (B) is prohibited by Lemma 6(c), the possible constellation at time $n$ is of type (G2). If $\varepsilon_{n}=1$, then the constellation at time $n+1$ is of type (G2) with $\varrho_{n+1}=\left|D_{n+1}^{(0)}\right|$. For balance at time $n+1$, the inequality

$$
\left|D_{n+1}^{(1)}\right|=\left|D_{n}^{(0)}+D_{n}^{(2)}\right| \leq\left|D_{n+1}^{(2)}\right|=\left|D_{n}^{(1)}\right|
$$

must hold, for if not, Lemma 6 (b) holds for $\mathcal{C}_{n+1}$. Thus $\mathcal{C}_{n+1}$ must be balanced with

$$
\left|D_{n+1}^{(0)}\right| \geq\left|D_{n+1}^{(2)}\right| \geq\left|D_{n+1}^{(1)}\right| \quad(\rightarrow \text { Case V }(\mathrm{G} 2)) .
$$

If $\varepsilon_{n}=2$, then the constellation at time $n+1$ is of type (G2) with $\varrho_{n+1}=$ $\left|D_{n+1}^{(1)}\right|$. For balance at time $n+1$, the inequality

$$
\left|D_{n+1}^{(0)}\right|=\left|D_{n}^{(0)}+D_{n}^{(2)}\right| \leq\left|D_{n+1}^{(2)}\right|=\left|D_{n}^{(1)}\right|
$$

must hold, for if not, Lemma 6 (b) holds for $\mathcal{C}_{n+1}$. Thus $\mathcal{C}_{n+1}$ must be balanced.

CASE III: $\left|D_{n}^{(1)}\right| \geq\left|D_{n}^{(0)}\right| \geq\left|D_{n}^{(2)}\right|$. Being balanced implies that $y$ lies between $A$ and $B$. Possible constellations are therefore (G1) and (B). Since the constellation of type (G1) is prohibited by Lemma $6(\mathrm{~d})$, the constellation must be of type (B). If $\varepsilon_{n}=1$, then the constellation at time $n+1$ is of type (G2) and easily seen to be balanced. If $\left|D_{n}^{(0)}+D_{n}^{(2)}\right| \geq\left|D_{n}^{(1)}\right|$, then

$$
\left|D_{n+1}^{(1)}\right| \geq\left|D_{n+1}^{(2)}\right| \geq\left|D_{n+1}^{(0)}\right| \quad(\rightarrow \text { Case IV }(\mathrm{G} 2))
$$

and if $\left|D_{n}^{(0)}+D_{n}^{(2)}\right| \leq\left|D_{n}^{(1)}\right|$, then

$$
\left|D_{n+1}^{(2)}\right| \geq\left|D_{n+1}^{(1)}\right| \geq\left|D_{n+1}^{(0)}\right| \quad(\rightarrow \text { Case II }(\mathrm{G} 2)) .
$$

If $\varepsilon_{n}=2$, then the constellation at time $n+1$ is also of type (G2) and easily seen to be balanced.

CASE IV: $\left|D_{n}^{(1)}\right| \geq\left|D_{n}^{(2)}\right| \geq\left|D_{n}^{(0)}\right|$. Being balanced implies that $y$ lies between $B$ and $C$. Possible constellations are therefore (G2) and (B). For both cases, if $\varepsilon_{n}=1$, then the constellation at time $n+1$ is of type (G2) and easily seen to be balanced with

$$
\left|D_{n+1}^{(2)}\right| \geq\left|D_{n+1}^{(0)}\right| \geq\left|D_{n+1}^{(1)}\right| \quad(\rightarrow \text { Case I }(\mathrm{G} 2)) .
$$

If $\varepsilon_{n}=2$, then the constellation at time $n+1$ is also of type (G2) and easily seen to be balanced. 
CASE V: $\left|D_{n}^{(0)}\right| \geq\left|D_{n}^{(2)}\right| \geq\left|D_{n}^{(1)}\right|$. Being balanced implies that $y$ lies between $A$ and $C$. Possible constellations are therefore (G1) and (G2). Suppose that the constellation at time $n$ is of type (G2). If $\varepsilon_{n}=1$, then the constellation at time $n+1$ is of type (G1) and easily seen to be balanced. If $\left|D_{n}^{(0)}+D_{n}^{(2)}\right| \geq\left|D_{n}^{(2)}\right|$, then $\left|D_{n+1}^{(1)}\right| \geq\left|D_{n+1}^{(0)}\right| \geq\left|D_{n+1}^{(2)}\right|$. But this shows that $\mathcal{C}_{n+1}$ is of type (G1) in Case III, which is not allowed. If $\left|D_{n}^{(1)}\right| \leq\left|D_{n}^{(0)}+D_{n}^{(2)}\right| \leq\left|D_{n}^{(2)}\right|$, then

$$
\left|D_{n+1}^{(0)}\right| \geq\left|D_{n+1}^{(1)}\right| \geq\left|D_{n+1}^{(2)}\right| \quad(\rightarrow \text { Case VI }(\mathrm{G} 1))
$$

and if $\left|D_{n}^{(0)}+D_{n}^{(2)}\right| \leq\left|D_{n}^{(1)}\right|$, then

$$
\left|D_{n+1}^{(0)}\right| \geq\left|D_{n+1}^{(2)}\right| \geq\left|D_{n+1}^{(1)}\right| \quad(\rightarrow \text { Case V }(\mathrm{G} 1)) .
$$

If $\varepsilon_{n}=2$, then the constellation at time $n+1$ is also of type (B) and easily seen to be balanced.

Suppose that the constellation at time $n$ is of type (G1). If $\varepsilon_{n}=1$, then the constellation at time $n+1$ is of type (B). For balance at time $n+1$, the inequality

$$
\left|D_{n+1}^{(1)}\right|=\left|D_{n}^{(0)}+D_{n}^{(2)}\right| \geq\left|D_{n+1}^{(2)}\right|=\left|D_{n}^{(1)}\right|
$$

must hold, for if not, Lemma 6 (c) holds for $\mathcal{C}_{n+1}$. Thus $\mathcal{C}_{n+1}$ must be balanced. Furthermore, it has

$$
\left|D_{n+1}^{(1)}\right| \geq\left|D_{n+1}^{(0)}\right| \geq\left|D_{n+1}^{(2)}\right| \quad(\rightarrow \text { Case III }(\mathrm{B}))
$$

if $\left|D_{n}^{(0)}+D_{n}^{(2)}\right| \geq\left|D_{n}^{(2)}\right|$, and

$$
\left|D_{n+1}^{(0)}\right| \geq\left|D_{n+1}^{(1)}\right| \geq\left|D_{n+1}^{(2)}\right| \quad(\rightarrow \text { Case VI }(\mathrm{B}))
$$

if $\left|D_{n}^{(0)}+D_{n}^{(2)}\right| \leq\left|D_{n}^{(2)}\right|$. Similarly, if $\varepsilon_{n}=2$, then the constellation at time $n+1$ is of type (G1). For balance at time $n+1$, the inequality

$$
\left|D_{n+1}^{(0)}\right|=\left|D_{n}^{(0)}+D_{n}^{(2)}\right| \geq\left|D_{n+1}^{(2)}\right|=\left|D_{n}^{(1)}\right|
$$

must hold, for if not, Lemma $6\left(\right.$ a) holds for $\mathcal{C}_{n+1}$. A contradiction. Thus $\mathcal{C}_{n+1}$ must be balanced.

CASE VI: $\left|D_{n}^{(0)}\right| \geq\left|D_{n}^{(1)}\right| \geq\left|D_{n}^{(2)}\right|$. Being balanced implies that $y$ lies between $A$ and $B$. Possible constellations are therefore (G1) and (B). Suppose that the constellation at time $n$ is of type (B). If $\varepsilon_{n}=1$, then the constellation at time $n+1$ is of type (G2) and easily seen to be balanced with

$$
\left|D_{n+1}^{(1)}\right| \geq\left|D_{n+1}^{(2)}\right| \geq\left|D_{n+1}^{(0)}\right| \quad(\rightarrow \text { Case IV }(\mathrm{G} 2))
$$

If $\varepsilon_{n}=2$, then the constellation at time $n+1$ is also of type (G2) and easily seen to be balanced. 
Suppose that the constellation at time $n$ is of type (G1). If $\varepsilon_{n}=1$, then the constellation at time $n+1$ is of type (B). For balance at time $n+1$, the inequality

$$
\left|D_{n+1}^{(1)}\right|=\left|D_{n}^{(0)}+D_{n}^{(2)}\right| \geq\left|D_{n+1}^{(0)}\right|=\left|D_{n}^{(2)}\right|
$$

must hold, for if not, Lemma 6 (c) holds for $\mathcal{C}_{n+1}$, contrary to weak convergence. Thus $\mathcal{C}_{n+1}$ must be balanced. If $\left|D_{n}^{(0)}+D_{n}^{(2)}\right| \leq\left|D_{n}^{(1)}\right|$, then $\left|D_{n+1}^{(2)}\right| \geq\left|D_{n+1}^{(1)}\right| \geq\left|D_{n+1}^{(0)}\right|$. But this type (B) in Case II is not allowed. Thus $\left|D_{n}^{(0)}+D_{n}^{(2)}\right| \geq\left|D_{n}^{(1)}\right|$ and

$$
\left|D_{n+1}^{(1)}\right| \geq\left|D_{n+1}^{(2)}\right| \geq\left|D_{n+1}^{(0)}\right| \quad(\rightarrow \text { Case IV }(\mathrm{B})) .
$$

Similarly, if $\varepsilon_{n}=2$, then the constellation at time $n+1$ is of type (G1). For balance at time $n+1$, the inequality

$$
\left|D_{n+1}^{(0)}\right|=\left|D_{n}^{(0)}+D_{n}^{(2)}\right| \geq\left|D_{n+1}^{(1)}\right|=\left|D_{n}^{(2)}\right|
$$

must hold, for if not, Lemma 6 (a) holds for $\mathcal{C}_{n+1}$. Thus $\mathcal{C}_{n+1}$ must be balanced. This completes the proof of (a).

The statement (b) follows by tracing four successive triples starting with an arbitrary $\mathcal{C}_{k}$ with $\varepsilon_{k}=1, \varepsilon_{k+1}=1, \varepsilon_{k+2}=1$ and $\varepsilon_{k+3}=1$.

If $\mathcal{C}_{n}$ has a bad constellation (see Definition 3.2) then it may happen that $\varrho_{n}<\varrho_{n+1}$; otherwise $\varrho_{n} \geq \varrho_{n+1}$. However, we have the following proposition.

Proposition 8. Suppose that the triple $\mathcal{C}_{n}=\left(r_{n}^{(0)} / q_{n}^{(0)}, r_{n}^{(1)} / q_{n}^{(1)}, r_{n}^{(2)} / q_{n}^{(2)}\right)$ at time $n$ has a bad constellation with $\varrho_{n}<\varrho_{n+1}$ and that the next bad constellation occurs at time $n+\tau$. Then there is an integer $l \leq \tau$ such that

$$
\varrho_{n+1} \leq 2 \varrho_{n}, \quad \varrho_{n+1}=\cdots=\varrho_{n+l}, \quad \varrho_{n} \geq \varrho_{n+l+1} \geq \cdots \geq \varrho_{n+\tau} .
$$

Proof. A bad constellation is a constellation of type (B) so that $C$ and $A$ are located on the same side of $y$. Remember that by Lemma 7 the triples generated by the algorithm are balanced. We consider several cases. Assume that $\mathcal{C}_{n}$ has a bad constellation which, as mentioned before, must be balanced.

CASE I: $\varrho_{n}=\left|D_{n}^{(1)}\right|$ and $\varepsilon_{n}=1$. By assumption, $\mathcal{C}_{n}$ is of type (B). We may assume that $B \leq y \leq A$ and $y \leq C$.

SubCASE Ia: $\left|D_{n}^{(1)}\right| \geq\left|D_{n}^{(0)}+D_{n}^{(2)}\right|$. Since $\varrho_{n}=\varrho_{n+1}=\left|D_{n}^{(1)}\right|, \varrho_{n+k}$ does not increase for $1 \leq k \leq \tau$ until a next bad constellation at time $\tau$.

Subcase Ib: $\left|D_{n}^{(1)}\right| \leq\left|D_{n}^{(0)}+D_{n}^{(2)}\right|$. In this case, $\mathcal{C}_{n+1}$ is of type (G2) with $\left|D_{n+1}^{(1)}\right| \geq\left|D_{n+1}^{(2)}\right| \geq\left|D_{n+1}^{(0)}\right|$. Clearly

$$
\varrho_{n}=\left|D_{n}^{(1)}\right| \leq \varrho_{n+1}=\left|D_{n+1}^{(1)}\right|=\left|D_{n}^{(0)}+D_{n}^{(2)}\right| \leq 2 \varrho_{n} .
$$


Whatever the value of $\varepsilon_{n+1}$, the next triple $\mathcal{C}_{n+2}$ is of type (G2) with

$$
\varrho_{n+2}=\left|D_{n+2}^{(2)}\right|=\left|D_{n+1}^{(1)}\right|=\varrho_{n+1} .
$$

Furthermore, we obtain

$$
\left|D_{n+1}^{(2)}\right| \geq\left|D_{n+2}^{(0)}\right|, \quad\left|D_{n+1}^{(2)}\right| \geq\left|D_{n+2}^{(1)}\right| .
$$

If $\varepsilon_{n+2}=2$, then $\mathcal{C}_{n+3}$ is, irrespective of $\varepsilon_{n+1}$, of type (G2) with

$$
\varrho_{n+3}=\left|D_{n+3}^{(1)}\right|=\left|D_{n+2}^{(2)}\right|=\varrho_{n+2} .
$$

Moreover, we have

$$
\left|D_{n+1}^{(2)}\right| \geq\left|D_{n+3}^{(0)}\right|, \quad\left|D_{n+1}^{(2)}\right| \geq\left|D_{n+3}^{(2)}\right|
$$

and $\left|D_{n+3}^{(1)}\right| \geq\left|D_{n+3}^{(2)}\right| \geq\left|D_{n+3}^{(0)}\right|$ since $\mathcal{C}_{n+3}$ must be balanced. This is exactly the same situation as $\mathcal{C}_{n+1}$. Thus we can repeat the same argument until we get $\varepsilon_{n+2 k}=1(k \geq 1)$ for the first time.

If $\varepsilon_{n+2 k}=1$, then $\mathcal{C}_{n+2 k+1}$ is also of type (G2) with

$$
\varrho_{n+2 k+1}=\left|D_{n+2 k+1}^{(0)}\right|=\left|D_{n+2 k}^{(2)}\right|=\varrho_{n+2 k}=\varrho_{n+1} .
$$

It follows inductively that the 2 nd and 3 rd maximal element of $\mathcal{C}_{n+j}$ in modulus is less than $\left|D_{n+1}^{(2)}\right|$ for $1 \leq j \leq 2 k+1$. In particular

$$
\left|D_{n+2 k-1}^{(2)}\right| \leq\left|D_{n+1}^{(2)}\right| \leq \varrho_{n+1} \text {. }
$$

Since $\mathcal{C}_{n+2 k+1}$ must be balanced, we have

$$
\left|D_{n+2 k+1}^{(1)}\right| \leq\left|D_{n+2 k+1}^{(2)}\right| .
$$

This inequality is equivalent to

$$
D_{n+2 k-1}^{(1)}+2 D_{n+2 k-1}^{(2)}+D_{n+2 k-1}^{(0)} \geq 0
$$

irrespective of $\varepsilon_{n+2 k-1}$. Thus

$$
\left|D_{n+2 k+1}^{(2)}+D_{n+2 k+1}^{(0)}\right| \leq\left|D_{n+2 k-1}^{(0)}+D_{n+2 k-1}^{(1)}+D_{n+2 k-1}^{(2)}\right| \leq\left|D_{n+2 k-1}^{(2)}\right| .
$$

Observe

$$
D_{n+2 k+1}^{(2)}= \begin{cases}D_{n+2 k-1}^{(2)} & \text { if } \varepsilon_{n+2 k-1}=1 \\ D_{n+2 k-1}^{(0)}+D_{n+2 k-1}^{(2)} & \text { if } \varepsilon_{n+2 k-1}=2 .\end{cases}
$$

Then, by (10), we have

$$
\varrho_{n+2 k+2}= \begin{cases}\left|D_{n+2 k-1}^{(2)}+D_{n+2 k-1}^{(0)}\right| & \text { if } \varepsilon_{n+2 k-1}=1, \\ \left|D_{n+2 k-1}^{(2)}\right| & \text { if } \varepsilon_{n+2 k-1}=2 .\end{cases}
$$

The triple $\mathcal{C}_{n+2 k+2}$ may have the next bad constellation (type (B)!). As a consequence, we have $\varrho_{n+2 k+2} \leq\left|D_{n+2 k-1}^{(2)}\right|$. Combining this with (9), it follows that $\varrho_{n+2 k+2} \leq \varrho_{n+1}$. Obviously $\varrho_{n+\tau} \leq \cdots \leq \varrho_{n+2 k+2} \leq \varrho_{n+1}$. 
CASE II: $\varrho_{n}=\left|D_{n}^{(1)}\right|$ and $\varepsilon_{n}=2$.

SubCASE IIa: $\left|D_{n}^{(1)}\right| \geq\left|D_{n}^{(0)}+D_{n}^{(2)}\right|$. Since $\varrho_{n+1}=\left|D_{n+1}^{(2)}\right|=\left|D_{n}^{(1)}\right|=\varrho_{n}$, there is no increase.

SubCASE IIb: $\left|D_{n}^{(1)}\right| \leq\left|D_{n}^{(0)}+D_{n}^{(2)}\right|$. Since $\left|D_{n+1}^{(0)}+D_{n+1}^{(2)}\right| \leq\left|D_{n}^{(1)}\right|$, $\varrho_{n+2}=\varrho_{n}$. Clearly $\varrho_{n+1}=\left|D_{n}^{(0)}+D_{n}^{(2)}\right| \leq 2 \varrho_{n}$.

CASE III: $\varrho_{n}=\left|D_{n}^{(2)}\right|$. This assumption implies that $\mathcal{C}_{n+1}$ is not balanced. Hence this case is impossible.

CASE IV: $\varrho_{n}=\left|D_{n}^{(0)}\right|$ and $\varepsilon_{n}=1$. For balance at time $n,\left|D_{n}^{(1)}\right| \geq\left|D_{n}^{(2)}\right|$. Then $\mathcal{C}_{n+1}$ faces the same situation as the triple at time $n+1$ in Case I. Repeating the same argument yields this case.

CASE V: $\varrho_{n}=\left|D_{n}^{(0)}\right|$ and $\varepsilon_{n}=2$. For balance at time $n,\left|D_{n}^{(1)}\right| \geq\left|D_{n}^{(2)}\right|$. Since $\left|D_{n}^{(0)}+D_{n}^{(1)}+D_{n}^{(2)}\right| \leq\left|D_{n}^{(0)}\right|$, we have $\varrho_{n+2} \leq \varrho_{n}$.

Verification of (H6). Let $n_{k}$ be the subsequence for which the triple $\mathcal{C}_{n_{k}}$ has a bad constellation. Then Proposition 8 gives (a) and (b) of (H6) with $C_{1}=2$. Condition (c) follows by Lemma 7 (b) with $l=4$. This completes the proof of Corollary 2 for the algorithm.

4. The algorithm of Brun [2]. Consider a three-dimensional simplex

$$
\Omega^{2+1}=\left\{\mathbf{b}=\left(b_{1}, b_{2}, b_{3}\right): 0 \leq b_{1} \leq b_{2} \leq b_{3}\right\} .
$$

Define a transformation $\widetilde{T}: \Omega^{2+1} \rightarrow \Omega^{2+1}$ obtained from

$$
\sigma \mathbf{b}=\left(b_{1}, b_{2}, b_{3}-b_{2}\right)
$$

by making necessary permutations of coordinates to have an image in $\Omega^{2+1}$. A transformation $T: \Delta \rightarrow \Delta$ is obtained by a commutative diagram $T \circ \pi=$ $\pi \circ \widetilde{T}$, where $\pi\left(b_{1}, b_{2}, b_{3}\right)=\left(b_{1} / b_{3}, b_{2} / b_{3}\right)$ is the quotient map and

$$
\Delta=\{(x, y): 0 \leq x \leq y \leq 1\} .
$$

Explicitly

$$
T(x, y)= \begin{cases}\left(\frac{x}{1-y}, \frac{x}{1-y}\right) & \text { if } y \leq 1 / 2, \\ \left(\frac{x}{y}, \frac{1-y}{y}\right) & \text { if } y \geq 1 / 2, x+y \leq 1, \\ \left(\frac{1-y}{y}, \frac{x}{y}\right) & \text { if } x+y \geq 1 .\end{cases}
$$


There is a natural partition

$$
\begin{aligned}
\Delta= & \Delta(1) \cup \Delta(2) \cup \Delta(3) \\
= & \{(x, y) \in \Delta: y \leq 1 / 2\} \cup\{(x, y) \in \Delta: y \geq 1 / 2 \text { and } x+y \leq 1\} \\
& \cup\{(x, y) \in \Delta: x+y \geq 1\}
\end{aligned}
$$

such that $T(\Delta(j))=\Delta$ for $j=1,2,3$. Set $\left(x_{n}, y_{n}\right)=T^{n}(x, y)$ for $n>0$ and $\left(x_{0}, y_{0}\right)=(x, y)$. Let $\varepsilon(x, y)=j$ if $(x, y) \in \Delta(j)$ for $1 \leq j \leq 3$ and define a coding $\left\{\varepsilon_{n}\right\}$ of the orbit by $\varepsilon_{n}=\varepsilon\left(x_{n}, y_{n}\right)$ and $\varepsilon_{0}=\varepsilon(x, y)$. Then, using the row vector $u_{1}=\left(x_{1}, y_{1}\right)$, the equation

$$
u_{0}=\theta(x, y) u_{1} M(x, y)
$$

defines the associated $3 \times 3$ matrices $M(x, y)$, where

$$
\theta(x, y)= \begin{cases}1 /\left(1+y_{1}\right) & \text { if } \varepsilon=1,2, \\ 1 /\left(1+x_{1}\right) & \text { if } \varepsilon=3 .\end{cases}
$$

Two-dimensional Brun's algorithm has three matrices $M(x, y)$ allowable:

$$
\left(\begin{array}{lll}
1 & 0 & 0 \\
0 & 1 & 1 \\
0 & 0 & 1
\end{array}\right) \text { if } \varepsilon_{0}=1, \quad\left(\begin{array}{lll}
1 & 0 & 0 \\
0 & 0 & 1 \\
0 & 1 & 1
\end{array}\right) \text { if } \varepsilon_{0}=2, \quad\left(\begin{array}{lll}
0 & 0 & 1 \\
1 & 0 & 0 \\
0 & 1 & 1
\end{array}\right) \text { if } \varepsilon_{0}=3 .
$$

Then three recursive equations are obtained:

$$
\begin{array}{llll}
q_{n+1}^{(0)}=q_{n}^{(0)}, & q_{n+1}^{(1)}=q_{n}^{(1)}+q_{n}^{(2)}, \quad q_{n+1}^{(2)}=q_{n}^{(2)} & \text { if } \varepsilon_{n}=1, \\
q_{n+1}^{(0)}=q_{n}^{(0)}, & q_{n+1}^{(1)}=q_{n}^{(2)}, \quad q_{n+1}^{(2)}=q_{n}^{(1)}+q_{n}^{(2)} & \text { if } \varepsilon_{n}=2, \\
q_{n+1}^{(0)}=q_{n}^{(2)}, & q_{n+1}^{(1)}=q_{n}^{(0)}, & q_{n+1}^{(2)}=q_{n}^{(1)}+q_{n}^{(2)} & \text { if } \varepsilon_{n}=3 .
\end{array}
$$

If we set $p_{n}^{(j)}:=p_{n}^{(j, 1)}$ and $r_{n}^{(j)}:=p_{n}^{(j, 2)}$, then the same recursive equations hold for these $p_{n}^{(j)}$ and $r_{n}^{(j)}$. The following facts are well known (see [4], [6] for example):

- $\mu$ is an $T$-invariant ergodic measure which is equivalent to Lebesgue measure.

- Brun's algorithm is weakly convergent almost everywhere.

And it is easy to verify

- $M(x, y)$ is spectrally $\mathrm{PV}$ on $\Delta(3)$ with a positive eigenvector.

We will verify (H6) only for $\varrho_{n}^{(2)}$. Similar results for $\varrho_{n}^{(1)}$ can be easily obtained. Set $D_{n}^{(j)}:=D_{n}^{(j, 2)}=q_{n}^{(j)} y-r_{n}^{(j)}$ and $\varrho_{n}:=\varrho_{n}^{(2)}=\max _{0 \leq j \leq 2}\left\{\left|D_{n}^{(j)}\right|\right\}$. 
Definition 4.1. A triple $\mathcal{C}_{n}=\left(r_{n}^{(0)} / q_{n}^{(0)}, r_{n}^{(1)} / q_{n}^{(1)}, r_{n}^{(2)} / q_{n}^{(2)}\right)$ generated by Brun's algorithm is said to have a bad constellation if $r_{n}^{(1)} / q_{n}^{(1)}$ and $r_{n}^{(2)} / q_{n}^{(2)}$ are on the same side of $y$ on the real line. Otherwise $\mathcal{C}_{n}$ is said to have a good constellation.

We know that $\varrho_{n+1}>\varrho_{n}$ only if $\mathcal{C}_{n}$ has a bad constellation.

Proposition 9. Suppose that the algorithm is weakly convergent at $(x, y)$. Then $\varrho_{n+1} \leq \varrho_{n}$ for all $n$.

Proof. First consider the following constellation $\mathcal{C}_{k}=(A, B, C)$ :

$$
B \leq y \leq A, y \leq C \quad(\text { or } B \geq y \geq A, y \geq C)
$$

with $\left|D_{k}^{(1)}\right| \leq\left|D_{k}^{(2)}\right|$. Then, whatever the value of $\varepsilon_{k}$, all fractions in $\mathcal{C}_{k+1}$ are on one side of $y$. By Lemma 4, we have no weak convergence at this point. This constellation $\mathcal{C}_{k}$ is said to be of prohibited type.

Suppose that $\mathcal{C}_{n}$ has a bad constellation. The proof proceeds by induction and dividing into cases.

CASE I: $\left|D_{n}^{(0)}\right| \leq\left|D_{n}^{(1)}\right|$ and $\left|D_{n}^{(0)}\right| \leq\left|D_{n}^{(2)}\right|$. Recursive equations (11) and (12) imply that the fraction $r_{n}^{(0)} / q_{n}^{(0)}$ never disappears as long as $\varepsilon_{n+k}$ takes the value in $\{1,2\}$ for $k \geq 0$. By weak convergence, it follows that there is a minimal integer $k \geq 0$ with $\varepsilon_{n+k}=3$. Then $\mathcal{C}_{n+k}$ is of prohibited type. Hence this case is impossible.

CASE II: $\left|D_{n}^{(1)}\right| \leq\left|D_{n}^{(0)}\right| \leq\left|D_{n}^{(2)}\right|$. If $\varepsilon_{n}=3$, then $\mathcal{C}_{n+1}$ is of prohibited type. If $\varepsilon_{n}=1$ or 2 , then $\mathcal{C}_{n+1}$ is as in Case I. This case is also impossible.

CASE III: $\left|D_{n}^{(2)}\right| \leq\left|D_{n}^{(0)}\right| \leq\left|D_{n}^{(1)}\right|$. If $\varepsilon_{n}=3$, then $\mathcal{C}_{n+1}$ is of prohibited type. If $\varepsilon_{n}=2$, then $\mathcal{C}_{n+1}$ is as in Case II. If $\varepsilon_{n}=1$, then $\mathcal{C}_{n+1}$ is as in Case III. This repetition of Case III eventually stops by weak convergence. Thus this case never happens.

CASE IV: $\varrho_{n}=\left|D_{n}^{(0)}\right|$.

SubCASE I: $\left|D_{n}^{(1)}+D_{n}^{(2)}\right| \leq\left|D_{n}^{(0)}\right|$. Then $\varrho_{n+1} \leq \varrho_{n}$.

SubCASE II: $\left|D_{n}^{(1)}+D_{n}^{(2)}\right| \geq\left|D_{n}^{(0)}\right|$. If $\varepsilon_{n}=3$, then $\mathcal{C}_{n+1}$ is of prohibited type. If $\varepsilon_{n}=2$, then $\mathcal{C}_{n+1}$ is as in Case II. If $\varepsilon_{n}=1$, then $\mathcal{C}_{n+1}$ is as in Case III. Hence this subcase is impossible.

Verification of (H6). There is no need to take a subsequence by Proposition 9. Thus this algorithm satisfies (H6), which completes the proof of Corollary 2.

Acknowledgements. The author thanks the referee for helpful comments. This research was partially supported by JSPS research fellowship. 


\section{References}

[1] A. Broise-Alamichel et Y. Guivarc'h, Exposants caractéristiques de l'algorithme de Jacobi-Perron et de la transformation associée, Ann. Inst. Fourier (Grenoble) 51 (2001), 565-686.

[2] V. Brun, Algorithmes euclidiens pour trois et quatre nombres, in: Treizième congrès des mathématiciens scandinaves (Helsinki, 1957), Mercators Tryckeri, Helsinki, 1958, $45-64$.

[3] M. W. Hirsch and S. Smale, Differential Equations, Dynamical Systems, and Linear Algebra, Academic Press, 1974.

[4] J. C. Lagarias, The quality of the Diophantine approximations found by the JacobiPerron algorithm and related algorithms, Monatsh. Math. 115 (1993), 299-328.

[5] F. Schweiger, Ergodic and Diophantine properties of algorithms of Selmer type, Acta Arith. 114 (2004), 99-111.

[6] —, Multidimensional Continued Fractions, Oxford Univ. Press, 2000.

[7] E. Selmer, Continued fractions in several dimensions, Nordisk Nat. Tidskr. 9 (1961), 37-43, 95 (in Norwegian).

Graduate School of Mathematical Sciences

University of Tokyo

3-8-1 Komaba, Meguro, Tokyo 153-8914, Japan

E-mail: nakaishi@ms.u-tokyo.ac.jp

Received on 11.3.2005

and in revised form on 25.7.2005 